\title{
On the Analysis of the Kronecker Product of Irreducible Representations of the Symmetric Group
}

\author{
By Ragy H. Makar
}

(Received 29th November, 1947.

Read 4th March, 1949.)

\section{Introduction}

The Kronecker product of two irreducible matrix representations $D(\lambda), D(\mu)$ of the symmetric group on $n$ letters, furnishes a representation of that group, which is, in general reducible. The question of what irreducible representations will appear in the analysis of such products has been dealt with by Prof. F. D. Murnaghan.' Indeed he has obtained the analysis of $D\left(n-p, \lambda_{2}, \ldots\right) \times D\left(n-q, \mu_{2}, \ldots\right)$, for the particular values, $p=1, q=1,2,3,4,5 ; p=2, q=2,3,4$; $p=3, q=3$, 4, applying a method which is a recurrence one, in the sense that to obtain such an analysis we have to look at some other analyses which come first in order.

In this short note, the same problem is dealt with, but in an entirely different way. Formulae are given which provide us with a direct method of obtaining the analysis of $D\left(n-p, \lambda_{2} \ldots\right) \times D(\mu)$, where $p=1,2,3$, but $(\mu)$ is any partition of $n$. The formulae, in fact, turn the problem into that of multiplication of Schur-functions ${ }^{2}$ ( $S$-functions) of different weights, by the $S$-functions, $\{1\},\{2\},\left\{1^{2}\right\},\{3\},\{2,1\},\left\{1^{3}\right\}$. Multiplication by $\{1\},\{2\},\left\{1^{2}\right\},\{3\}$, is governed by very simple rules stated in reference (3), pp. 480-481. A similar rule may be added for the multiplication by $\left\{1^{3}\right\}$. Multiplication by $\{2,1\}$ can be performed by a very rapid method given in reference (1), pp. 308-309.

\section{S-functions and notation.}

The expression

$$
\{\lambda\}=\left\{\lambda_{1}, \lambda_{2}, \ldots, \lambda_{\kappa}\right\}=\frac{1}{n !} \sum_{(a)} n_{(a)} \chi_{(a)}^{(\lambda)} s_{1}^{a_{1}} s_{2}^{\alpha_{2}} \ldots s_{n}^{\alpha},
$$

is called the $S$-function corresponding to the partition $(\lambda)=\left(\lambda_{1}, \lambda_{2}, \ldots, \lambda_{\kappa}\right)$ of $n$, and is said to be of weight $n$. It is shown that ${ }^{3}$

$$
p \frac{\partial\{\lambda\}}{\partial S_{p}}=\sum_{i=1}^{\kappa}\left\{\lambda_{1}, \lambda_{2}, \ldots, \lambda_{i}-p, \ldots, \lambda_{\kappa}\right\}
$$

1 See reference (4) at the end.

2 Reference (2), chapter VI.

3 See reference (3), p. 461. 
where the $S$-functions on the right-hand side are of weight $n-p$.

On a second differentiation this gives

$$
p q \frac{\hat{\partial}^{2}\{\lambda\}}{\partial s_{p} \partial s_{q}}=\sum_{i=1}^{\kappa} \sum_{\delta=1}^{\kappa}\left\{\lambda_{1}, \lambda_{2}, \ldots, \lambda_{i}-p, \ldots, \lambda_{j}-q, \ldots, \lambda_{\kappa}\right\}
$$

and so on.

This justifies the use of the following notation:-

$$
\begin{array}{rlrl}
p \frac{\partial\{\lambda\}}{\partial s_{p}} & =\left\{\Lambda_{p}^{\prime}\right\}, p=1,2,3, & \frac{\partial^{2}\{\lambda\}}{\partial s_{1}{ }^{2}}=\left\{\Lambda^{\prime}{ }_{11}\right\} \\
2 \frac{\partial^{2}\{\lambda\}}{\partial s_{2} \partial s_{1}}=\left\{\Lambda_{21}^{\prime}\right\} & \frac{\partial^{3}\{\lambda\}}{\partial s_{1}{ }^{3}}=\left\{\Lambda_{111}^{\prime}\right\} .
\end{array}
$$

We also assume that:-

$\left\{\Lambda_{p}\right\}, p=1,2,3$, is obtained from $\left\{\lambda_{1}, \lambda_{2}, \ldots, \lambda_{k}\right\}$ by subtracting $p$ in all possible ways,

$\left\{\Lambda_{p q}\right\}, p=1,2, q=1$, is obtained by subtracting $(p, q)$ in all possible ways,

$\left\{\Lambda_{111}\right\}$ is obtained by subtracting $(1,1,1)$ in all possible ways

The following identities are then written immediately:

$$
\begin{array}{ll}
\left\{\Lambda_{p}^{\prime}\right\}=\left\{\Lambda_{p}\right\}, p=1,2,3, \quad\left\{\Lambda^{\prime}\right\}=\left\{2 \Lambda_{11}+\Lambda_{2}\right\} \\
\left\{\Lambda^{\prime}{ }_{21}\right\}=\left\{\Lambda_{21}+\Lambda_{3}\right\}, \quad & \left\{\Lambda^{\prime}{ }_{111}\right\}=\left\{6 \Lambda_{11}+3 \Lambda_{21}+\Lambda_{3}\right\}
\end{array}
$$

Formulae for the analysis

$$
\begin{aligned}
& \text { (I) } D(n-1,1) \times D(\lambda)=\Sigma D(\mu)-D(\lambda) \text {, } \\
& \left\{\Lambda_{1}\right\}\{1\} \quad=\Sigma\{\mu\} \text {. } \\
& \text { (II) } D(n-2,2) \times D(\lambda)=\Sigma D(\mu)+\Sigma D(\nu)-\Sigma D(\gamma) \text {, } \\
& \left\{\Lambda_{11}+\Lambda_{2}\right\}\{2\} \quad=\Sigma\{\mu\} \\
& \left\{\Lambda_{11}\right\}\left\{1^{2}\right\}=\Sigma\{\nu\}, \\
& \left\{\Lambda_{1}\right\}\{1\} \quad=\Sigma\{\gamma\} \text {. } \\
& \text { (III) } D\left(n-2,1^{2}\right) \times D(\lambda)=\Sigma D(\mu)+\Sigma D(\nu)-\Sigma D(\gamma)+D(\lambda) \text {, } \\
& \left\{\Lambda_{11}\right\}\{2\} \quad=\Sigma\{\mu\} \text {, } \\
& \left\{\Lambda_{11}+\Lambda_{2}\right\}\left\{1^{2}\right\} \quad=\Sigma\{\nu\} \text {, } \\
& \left\{\Lambda_{1}\right\}\{1\}=\Sigma\{\gamma\} \text {. }
\end{aligned}
$$




$$
\begin{aligned}
& \text { (IV) } D(n-3,3) \times D(\lambda)=\Sigma D(\mu)+\Sigma D(\nu)+\Sigma D(\epsilon)-\Sigma D(\gamma) \\
& -\Sigma D(\delta) \\
& \left\{\Lambda_{111}+\Lambda_{21}+\Lambda_{3}\right\}\{3\}=\Sigma\{\mu\}, \quad\left\{\Lambda_{11}+\Lambda_{2}\right\}\{2\}=\Sigma\{\gamma\}, \\
& \left\{2 \Lambda_{111}+\Lambda_{21}\right\}\{2,1\} \quad=\Sigma\{\nu\}, \quad\left\{\Lambda_{11}\right\}\left\{1^{2}\right\}=\Sigma\{\delta\}, \\
& \left\{\Lambda_{111}\right\}\left\{1^{3}\right\} \quad=\Sigma\{\epsilon\} \text {. } \\
& \text { (V) } D(n-3,2,1) \times D(\lambda)=\Sigma D(\mu)+\Sigma D(\nu)+\Sigma D(\epsilon)-\Sigma D(\gamma) \\
& \left\{2 \Lambda_{111}+\Lambda_{21}\right\}\{3\}=\Sigma\{\mu\}, \quad \cdot\left\{2 \Lambda_{11}+\Lambda_{2}\right\}\{2\}=\Sigma\{\gamma\}, \\
& \left\{4 \Lambda_{111}+2 \Lambda_{21}+\Lambda_{3}\right\}\{2,1\}=\Sigma\{\nu\}, \quad\left\{2 \Lambda_{11}+\Lambda_{2}\right\}\left\{1^{2}\right\}=\Sigma\{\delta\}, \\
& \left\{2 \Lambda_{111}+\Lambda_{21}\right\}\left\{1^{3}\right\} \quad=\Sigma\{\epsilon\}, \quad\left\{\Lambda_{1}\right\}\{1\} \quad=\Sigma\{\xi\} \text {. } \\
& \text { (VI) } D\left(n-3,1^{3}\right) \times D(\lambda)=\Sigma D(\mu)+\Sigma D(\nu)+\Sigma D(\epsilon)-\Sigma D(\gamma) \\
& \left\{\Lambda_{111}\right\}\{3\} \quad=\Sigma\{\mu\}, \quad\left\{\Lambda_{11}\right\}\{2\} \quad=\Sigma\{\gamma\}, \\
& \left\{2 \Lambda_{111}+\Lambda_{21}\right\}\{2,1\} \quad=\Sigma\{v\}, \quad\left\{\Lambda_{11}+\Lambda_{2}\right\}\left\{1^{2}\right\}=\Sigma\{\delta\}, \\
& \left\{\Lambda_{111}+\Lambda_{21}+\Lambda_{3}\right\}\left\{1^{3}\right\}=\Sigma\{\epsilon\}, \quad\left\{\Lambda_{1}\right\}\{1\}=\Sigma\{\xi\} .
\end{aligned}
$$

It is sufficient to establish formulae (VI); the others are established in a similar manner. We therefore prove that:

$$
\begin{aligned}
\chi_{(a)}^{\left(n-3,1^{3}\right)} \times \chi_{(a)}^{(\lambda)}=\sum_{(\mu)} \chi_{(a)}^{(\mu)}+\sum_{(v)} \chi_{(a)}^{(p)}+\sum_{(e)} \chi_{(a)}^{(\epsilon)} & -\sum_{(\gamma)} \chi_{(a)}^{(\gamma)}-\sum_{(\delta)} \chi_{(a)}^{(\delta)} \\
& +\sum_{(\xi)} \chi_{(a)}^{(\xi)}-\chi_{(a)^{\prime}}^{(\lambda)}
\end{aligned}
$$

for all classes $(a)$ of the symmetric group.

We note that ${ }^{1}$

$$
\begin{aligned}
\chi_{(a)}^{\left(n-3,1^{3}\right)} & =\frac{1}{8}\left(a_{1}-1\right)\left(a_{1}-2\right)\left(a_{1}-3\right)-\left(a_{1}-1\right) a_{2}+\alpha_{3} \\
& =\frac{1}{6} a_{1}\left(a_{1}-1\right)\left(a_{1}-2\right)-a_{1} a_{8}+a_{3}-\frac{1}{2} a_{1}\left(a_{1}-1\right)+a_{2}+a_{1}-1 .
\end{aligned}
$$

(i) The coefficient of $s_{1}{ }^{a_{1}} s_{2}{ }^{a_{2}} \ldots s_{n}{ }^{a_{n}}$ in $\left\{\Lambda_{111}^{\prime}\right\} . s_{1}{ }^{3}$ is by (2) the coefficient of $s_{1}{ }^{a}{ }^{-3} s_{2}{ }^{a}{ }_{2} \ldots s_{n}{ }^{a}{ }_{n}$ in $\frac{\partial^{3}\{\lambda\}}{\partial s_{1}{ }^{3}}$, and by (1) this is

$$
a_{1}\left(a_{1}-1\right)\left(a_{1}-2\right) n_{(a)} \chi_{(a)}^{(\lambda)} / n !
$$

Since ${ }^{2}$

$$
s_{1}^{3}=\{3\}+2\{2,1\}+\left\{1^{3}\right\}
$$

we write

$$
\left\{\Lambda^{\prime}{ }_{111}\right\}\left[\{3\}+2\{2,1\}+\left\{1^{3}\right\}\right]=\Sigma\left\{\mu^{\prime}\right\} \text {, }
$$

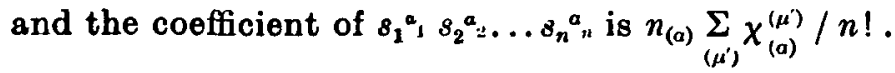

Reference (2), p. 139.

2 Reference (2), p. 86. 
Thus:

$$
\frac{1}{8} a_{1}\left(a_{1}-1\right)\left(a_{1}-2\right) \chi_{(a)}^{(2)}=\frac{1}{6} \sum_{\left(\mu^{\prime}\right)} \chi_{(a)}^{\left(\mu^{\prime}\right)}
$$

(ii) The coefficient of $s_{1}{ }^{a_{1}} s_{2}{ }^{a}{ }_{2} \ldots s_{n}{ }_{n}{ }_{n}$ in $\left\{\Lambda^{\prime}{ }_{21}\right\} s_{1} s_{2}$ is that of $s_{1}{ }^{a_{1}-1} s_{2}{ }^{a_{2}-1} \ldots s_{n}{ }^{a_{n}}$ in $2 \frac{\partial^{2}\{\lambda\}}{\partial s_{2} \hat{\partial} s_{1}}$, which is $2 a_{1} a_{2} n_{(a)} \chi_{(a)}^{(\lambda)} / n !$

Since

$$
s_{1} s_{2}=\{3\}-\left\{1^{3}\right\}
$$

we find

$$
a_{2} a_{2} \chi_{(a)}^{(2)}=\frac{1}{2} \Sigma_{\left(v^{\prime}\right)} \chi_{(a)}^{\left(\nu^{\prime}\right)}, \quad\left\{\Lambda_{21}^{\prime}\right\}\left[\{3\}-\left\{1^{3}\right\}\right]=\Sigma\left\{v^{\prime}\right\}
$$

(iii) The coefficient of $s_{1}{ }_{1}{ }_{1} s_{2}{ }_{2}{ }_{2} \ldots s_{n}{ }^{a} n$ in $\left\{\Lambda^{\prime}{ }_{3}\right\} . s_{3}$ is that of

$$
\begin{gathered}
s_{1}^{a} s_{2} s_{2}^{a} s_{3} a_{3}-1 \ldots s_{n}^{a} \text { in } 3 \frac{\partial\{\lambda\}}{\partial s_{3}}, \text { which is } a_{3} \chi_{(\alpha)}^{(l)} / n ! \\
s_{3}=\{3\}-\{2,1\}+\left\{1^{3}\right\},
\end{gathered}
$$

so that

$$
\alpha_{3} \chi_{(a)}^{(\alpha)}=\underset{\left(\epsilon^{\prime}\right)}{\mathcal{I}} \sum_{(a)}^{\left(\epsilon^{\prime}\right)}, \quad\left\{\Lambda_{3}^{\prime}\right\}\left[\{3\}-\{2,1\}+\left\{1^{3}\right\}\right]=\Sigma\left\{\epsilon^{\prime}\right\}
$$

so that

$$
s_{1}^{2}=\{2\}+\left\{1^{2}\right\}
$$

$$
\frac{1}{2} \alpha_{1}\left(\alpha_{1}-1\right) \chi_{(a)}^{(\lambda)}=\underset{\left(\gamma^{\prime}\right)}{\frac{1}{2}} \chi_{(a)}^{\left(\gamma^{\prime}\right)}, \quad\left\{\Lambda_{11}^{\prime}\right\}\left[\{2\}+\left\{1^{2}\right\}\right]=\Sigma\left\{\gamma^{\prime}\right\}
$$

$$
s_{2}=\{2\}-\left\{1^{2}\right\}
$$

so that

$$
\begin{array}{lll}
a_{2} \chi_{(a)}^{(\lambda)}=\underset{\left(\delta^{\prime}\right)}{\frac{1}{2}} \chi_{(a)}^{\left(\delta^{\prime}\right)}, & \left\{\Lambda_{2}^{\prime}\right\}\left[\{2\}-\left\{1^{2}\right\}\right] & =\Sigma\left\{\delta^{\prime}\right\} \\
a_{1} \chi_{(a)}^{(\lambda)}=\underset{\left(\xi^{\prime}\right)}{\chi_{(a)}^{\left(\xi^{\prime}\right)},} & \left\{\Lambda_{1}^{\prime}\right\}\{1\} & =\Sigma\left\{\xi^{\prime}\right\} .
\end{array}
$$

Adding up, we have

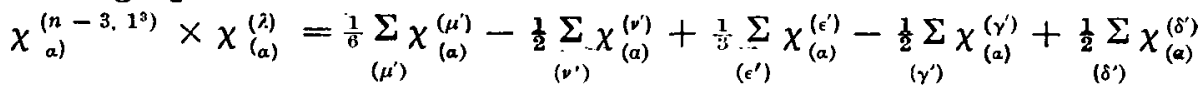

$$
\begin{aligned}
& +\underset{\left(\xi^{\prime}\right)}{\Sigma} \chi_{(\alpha)}^{\left(\xi^{\prime}\right)}-\chi_{(\alpha)}^{(\lambda)}
\end{aligned}
$$

Using the identities (4), we see that the expression on the right-hand side may be simply written as the expression on the right-hand side of (5).

To establish the other formulae we only note that the following characters can be written as: 


$$
\begin{aligned}
\chi_{(a)}^{(n-1,1)} & =a_{1}-1, \\
\chi_{(a)}^{(n-2,2)} & =\frac{1}{2} a_{1}\left(a_{1}-1\right)+a_{2}-1, \\
\chi_{(a)}^{(n-2,1=)} & =\frac{1}{2} a_{1}\left(a_{1}-1\right)-a_{2}-a_{1}+1, \\
\chi_{(a)}^{(n-3,3)} & =\frac{1}{6} a_{1}\left(a_{1}-1\right)\left(a_{1}-2\right)+a_{1} a_{2}+a_{2}-\frac{1}{2} a_{1}\left(a_{1}-1\right)-a_{2}, \\
\chi_{(a)}^{(n-3,2,1)} & =\frac{1}{3} a_{1}\left(a_{1}-1\right)\left(a_{1}-2\right)-a_{3}-a_{1}\left(a_{1}-1\right)+a_{1} .
\end{aligned}
$$

\section{Application of the formulae}

The simplicity of the formulae is seen through working a few examples. They give analyses of $D\left(n-p, \lambda_{2}, \ldots\right) \times D\left(n-q, \mu_{2} \ldots\right)$, $p=1,2,3, q$ unrestricted, which are valid for all symmetric groups. They also give the analysis of the Kronecker product of any irreducible representation of a given symmetric group, by one or other of certain irreducible representations of that group.

It is sufficient to record here two examples which have been worked out applying formulae (II) and (VI). If we write ( $\lambda$ ) for $D(\lambda)$, these analyses are:

$$
\begin{aligned}
& (n-2,2) \times\left(n-8,4^{2}\right)=(n-6,4,2)+(n-7,5,2)+2(n-7,4,3) \\
& +(n-7,4,2,1)+\left(n-7,3^{2}, 1\right)+(n-8,6,2)+2(n-8,5,3) \\
& +(n-8,5,2,1)+3\left(n-8,4^{2}\right)+3(n-8,4,3,1)+\left(n-8,4,2^{2}\right) \\
& +\left(n-8,3^{2}, 1^{2}\right)+(n-9,6,3)+2(n-9,5,4)+2(n-9,5,3,1) \\
& +2\left(n-9,4^{2}, 1\right)+(n-9,4,3,2)+\left(n-9,4,3,1^{2}\right)+(n-10,6,4) \\
& +(n-10,5,4,1)+\left(n-10,4^{2}, 2\right) . \\
& \left(12,1^{3}\right) \times(9,6)=(10,4,1)+\left(10,3,1^{2}\right)+(9,6)+2(9,5,1) \\
& +(9,4,2)+2\left(9,4,1^{2}\right)+\left(9,3,1^{3}\right)+2(8,6,1)+(8,5,2) \\
& +3\left(8,5,1^{2}\right)+(8,4,2,1)+\left(8,4,1^{3}\right)+\left(7^{2}, 1\right)+(7,6,2) \\
& +2\left(7,6,1^{2}\right)+(7,5,2,1)+\left(7,5,1^{3}\right)+\left(6^{2}, 1^{3}\right) .
\end{aligned}
$$

In deriving $\left\{\Lambda_{1}\right\},\left\{\Lambda_{11}\right\},\left\{\Lambda_{2}\right\}, \ldots$, as well as in performing the multiplication of $S$-functions, we apply the rules given in reference (2), pp. 98-99, to retain the descending order of parts of a partition.

In the second example $(\lambda)$ is a partition of two parts, so that $\left\{\Lambda_{111}\right\}$ does not exist.

\section{REFERENCES.}

(1) A. C. Aitken : "The monomial expansion of determinantal symmetric functions," Proc. Roy. Soc. Edinburgh, (A), 61 (1943).

(2) D. E. Lit tlewood: "The theory of group characters and matrix representations of groups," (Oxford, 1940).

(3) F. D. Murnaghan: "On the representations of the symmetric group," American Journ. of Math., 59 (1937).

(4) - : "The analysis of the Kronecker product of irreducible representations of the symmetric group," ibid., 60 (1938).

\section{The UnIVersity,}

abbassia, CaIro,

EgYPT. 